\title{
Licófitas e Samambaias no Cerrado do Leste do Maranhão, Brasil
}

\author{
Guilherme Sousa da Silvaa, Domingos Lucas dos Santos Silvab, Regigláucia Rodrigues de

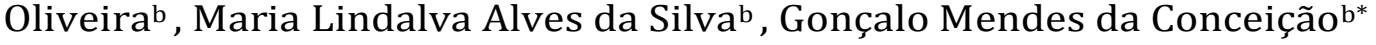 \\ a Laboratório de Biologia Vegetal, Centro de Estudos Superiores de Caxias, Caxias, 65604-380, Brasil. \\ b Programa de Pós-Graduação em Biodiversidade, Ambiente e Saúde, Universidade Estadual do Maranhão, Caxias, 65604-380, \\ Brasil,*doutorgoncalo@gmail.com
}

Recebido: 16 janeiro 2017/ Aceito: 24 fevereiro 2017 / Publicado online: 23 maio 2017

\begin{abstract}
Resumo
O Cerrado é um bioma estratégico para o Brasil, em virtude de sua diversidade e fonte para a economia brasileira. As (Lycophyta) Licófitas e (Monilophyta) Samambaias do Cerrado apresentam uma biodiversidade significativa de espécies, apesar dos poucos estudos para este domínio fitogeográfico. Essa pesquisa objetivou realizar um levantamento e caracterizar morfologicamente as espécies de Licófitas e samambaias ocorrentes em fragmentos vegetacionais do cerrado no município de São João do Sóter, Maranhão. Realizaram-se quatro excursões à área de estudo entre o período de outubro de 2015 a janeiro de 2016. O material botânico foi coletado, herborizado e identificado. Foram registradas 58 espécimes, distribuídos em 19 espécies, 14 gêneros e 11 famílias, sendo registrada uma nova ocorrência para o estado, a Cyclodium meniscioides C. Presl. O número de espécies de Licófitas e Samambaias registradas na área de estudo, até o momento, é o maior para o Maranhão.
\end{abstract}

Palavras-chave: Cyclodium, Fitofisionomias, Levantamento, Samambaias.

\section{Lycophytes and Ferns in the Cerrado of Eastern, Maranhão, Brazil}

\begin{abstract}
The Cerrado biome is one strategic for Brazil, due to your diversity and source for the Brazilian economy. The (Lycophyta) Lycophytes and (Monilophyta) Ferns of the Cerrado biodiversity species presente a asignificant, in spite of the few studies to this domain forest. This research aimed to perform a survey of the species and to characterize morphologically the species of Lyophytes and ferns occurring in vegetative fragments of Cerrado in the municipaly of São João do Sóter, Maranhão, characterized with diferente mosaics of Cerrado. There were four trips to the study area from october 2015 to january 2016 . The material was collected, herborized, identified and carried out taxonomic analyses. A total of 58 specimens where recorded, distributed in 19 species, 14 genera and 11 families, being registered a new occurrence for the state of Maranhão in the Northeast region, Cylodium meniscioides (Willd.) C. Presl. The number of species of Lyophytes and Ferns recorded in the study area, so far, is the largest for Maranhão.
\end{abstract}

Keywords: Cyclodium, Phytophysiognomies, Lifting, Ferns.

\section{Introdução}

As pteridófitas (Licófitas e Samambaias) apresentam uma gama de adaptações quanto ao substrato, forma de vida e ambientes preferenciais, exibindo uma diversidade de formas e variações de tamanho (Windisch, 1992; Moran, 2008). Licófitas e Samambaias são termos que se referem aos grupos de plantas vasculares sem flores e sementes, que se reproduzem por esporos (Zuquim et al., 2008; Prado et al., 2015).

O termo Pteridófita, que designa uma divisão das plantas vasculares, tem ficado em desuso devido aos estudos filogenéticos moleculares que apontam para a evolução distinta dos grupos que a compõem: Lycophyta (licófitas) e Monilophyta (samambaias), as quais são duas linhagens evolutivas distintas, separando o grupo Lycophyta das demais plantas vasculares, as Euphyllophyta (Pryer et al., 2004; Smith et al., 2008)

As licófitas caracterizam-se pela presença de microfilo e incluem três famílias: Lycopodiaceae, Selaginellaceae e Isoetaceae. A linhagem das Samambaias (Monilophyta) caracteriza-se por apresentar megafilos e vascularização distinta com o protoxilema confinado a lobos do cordão do xilema (Pryer et al., 2004), distribuídas nas classes Psilotopsida, Equisetopsida, Marattiopsida e Polypodiopsida, com cerca de 11.000 espécies distribuídas em 37 famílias (Smith et al., 2006).

Existem, aproximadamente, 1.300 espécies de Licófitas e 12.240 espécies de Samambaias para o mundo de acordo com Moran (2008), o que representa menos de $1 \%$ de todas as plantas vasculares (Smith et al., 2006).

Para o Brasil, estima-se a ocorrência de 1.253 espécies, 
destas, 1.111 espécies pertencem as Samambaias e 142 as Licófitas (Prado et al., 2015), incluindo 77 novos taxons quando comparado ao último levantamento realizado por Prado e Sylvestre (2010). Segundo a Flora do Brasil 2020 (2017), há ocorrência de 37 famílias, distribuídas em 152 gêneros e 1.313 espécies para o Brasil e, no estado do Maranhão, a ocorrência 20 famílias, 40 gêneros e 77 espécies.

O Cerrado contém um terço da biodiversidade brasileira e cerca de 5\% da flora e fauna mundial (Klink e Machado, 2005). As Samambaias e Licófitas no Cerrado apresentam um número significativo de espécies que ocorre em Matas de galeria, remanescentes de Matas mesófilas, Matas secas semidecíduas e Cerrados, apesar dos poucos estudos para este domínio fitogeográfico (Prado, 1998).

Para o Maranhão, existem estudos de Licófitas e Samambaias bastante representativos. Entretanto, os inventários são escassos quando se trata do Cerrado. Nesse contexto, essa pesquisa objetivou realizar um levantamento das espécies e caracterizar morfologicamente as Licófitas e Samambaias em fragmentos vegetacionais do Cerrado, no estado do Maranhão, Brasil.

\section{Material e Métodos}

A área de estudo foi o município de São João do Sóter ( $5^{\circ}$ 6' $28^{\prime \prime}$ S e $43^{\circ} 48^{\prime} 34^{\prime \prime} \mathrm{W}$ ), no estado do Maranhão, pertencente ao domínio fitogeográfico do Cerrado, em ambientes antropizados. A coleta do material ocorreu em três fragmentos vegetacionais pertencentes ao município: Povoado Pedras, Povoado Redondo e Centro Urbano. As áreas são remanescentes florestais com florestas semidecidual, apresentando corpos lóticos e lênticos, com uma predominância de babaçuais e árvores altas formando matas de galeria. O município se estende por $1.439,1 \mathrm{~km}^{2}$ e conta com 17.104 habitantes, com densidade demográfica 11,9 habitantes por $\mathrm{km}^{2}$ no território, estando situado a 108 metros de altitude a 400km da capital, São Luís (IBGE, 2012).

Foram realizadas quatro excursões mensais, no período de outubro de 2015 à janeiro de 2016, na área de estudo para coleta do material botânico, percorrendo as diferentes fitofisionomias como Cerrado limpo, Cerrado sujo, Mata de Galeria e Floresta Estacional Semidecidual dos ambientes preferenciais das Samambaias e Licófitas, através de caminhadas aleatórias. $\mathrm{O}$ material botânico foi coletado com o auxílio de tesoura de poda e desplantador, acondicionado em saco de plástico e etiquetados de acordo com a sequência da coleta, seguindo a metodologia de Fidalgo e Bononi (1989). Foram anotados: data e local da coleta, o nome do coletor, as características físicas do ambiente, o hábito da planta, características da planta, cor e textura das folhas, ausência ou não de esporos, disposição dos soros e outras características.

Para identificação, a classificação adotada para as Samambaias seguiu-se Pryer et al., (2004), descrita por Smith et al., (2008), enquanto que para as Licófitas, seguiu o sistema proposto por Kramer e Green (1990). Duplicadas foram enviadas para o Herbáreo NX para Coleção Zoobotânica James Alexander Ratter, da UNEMAT, Campus Universitário de Nova Xavantina/MT, para identificação e/ou confirmação dos mesmos. Após a identificação, foram obtidas a distribuição geográfica e dos domínios fitogeográficos em análise de dados encontrados em literatura pertinente e consulta ao site da Flora do Brasil 2020 (2017), adicionados ao material examinado e descrição breve das espécies amostradas, constituindo análises taxonômicas.

Os espécimes vegetais foram herborizados e depositados no Herbário Prof. Aluízio Bittencourt/HABIT, da Universidade Estadual do Maranhão/UEMA.

\section{Resultados e Discussão}

Foram encontrados 58 espécimes de Monilophyta, distribuídos em 19 espécies, 14 gêneros e 11 famílias, sendo apenas uma família de Licófitas (Selaginellaceae) (Tabela 1). Uma nova ocorrência, não catalogada na Flora do Brasil 2020 (2017) e levantamentos de Pteridófitas para o Estado do Maranhão, foi registrada: Cyclodium meniscioides (Willd.) C. Presl.

O hábito de vida mais frequente foi o de erva $(84,2 \%)$, ocupando em maior predominância substrato terrícola $(63,1 \%)$. As Samambaias e Licófitas são um importante grupo dentro da comunidade de plantas herbáceas das florestas tropicais compreendendo até $54 \%$ de todas as espécies de ervas amostradas (Costa, 2004). Quanto à predominância de espécies terrícolas, conforme Gonzatti et al. (2014), deve-se as características do ambiente no qual as espécies estão se desenvolvendo. Além disso, há uma variedade de espécies de Samambaias e Licófitas que apresentam preferência pelo substrato terrestre, independente do domínio fitogeográfico (Forsthofer e Athayde Filho, 2012), pois estas espécies não apresentam estruturas adaptativas em sua morfologica e/ou fisiologia para ocuparem outros tipos de ambientes (Goetz et al., 2012).

As espécies coletadas também são descritas em todos os trabalhos de Licófitas e Monilófitas publicados para o Maranhão, percebendo-se que o número de espécies citadas aumenta com o decorrer dos anos, segundo os levantamentos de Bastos e Cutrim (1999); Fernandes et al. (2007); Fernandes et al. (2010); Conceição e Rodrigues (2010); Conceição e Ruggieri (2010). Através do presente trabalho as espécies amostradas têm ampla distribuição no Maranhão, e principalmente na região Leste Maranhense onde os estudos citados foram realizados.

A presente pesquisa registrou o maior número de espécies coletadas para o Maranhão, quando comparada com estudos acima citados sobre a Pteridoflora. No entanto, conforme estimativas de Mendonça et al. (2008), no Cerrado brasileiro há a ocorrência de 385 espécies de Samambaias e Licófitas. Assim, esse estudo registrou a ocorrência de 4,9\% das espécies no Leste do Maranhão. Esta baixa riqueza das espécies, pode ser justificada pelos poucos estudos realizados na região, que com a ausência de estudos não há informações claras sobre a riqueza e diversidade desse grupo vegetal, além da conservação das áreas florestais, no qual grande parte das áreas coletadas está sendo destinadas a atividades agrícolas. 
Tabela 1. Caracterização morfológica, Distribuição geográfica e Fitogeográficas das espécies de Licófitas e Samambaias coletadas no Município de São João do Sóter, Maranhão.

\begin{tabular}{|c|c|c|c|c|}
\hline Família & Espécie & Hábito de Vida & Distribuição Geográfica & Domínios Fitogeográficos \\
\hline Blechnaceae & $\begin{array}{l}\text { Telmatoblechnum serrulatum (Rich.) } \\
\text { Perrie, D. J. Ohlsen \& Brownsey, Taxon } \\
\text { 63(4): 755. 2014. }\end{array}$ & Erva - Terrícola & $\begin{array}{l}\text { AP, RR, AM, PA, RO; PI, PB, BA, MA; MT, GO, DF, MS; MG, ES, RJ, SP; PR, } \\
\text { SC, RS }\end{array}$ & $\begin{array}{l}\text { Amazônia, Cerrado, Mata } \\
\text { Atlântica }\end{array}$ \\
\hline Cyatheacea & $\begin{array}{l}\text { Cyathea delgadii Sternb. Vers. Fl. } \\
\text { Vorwelt } 1: 47 \text {, t. B, } 1820 .\end{array}$ & $\begin{array}{l}\text { Árvore - } \\
\text { Terrícola }\end{array}$ & $\begin{array}{l}\text { AC, AM, RO, RR; BA, CE, PE, PI; DF, GO, MS, MT; ES, MG, RJ, SP; PR, RS, } \\
\text { SC }\end{array}$ & $\begin{array}{l}\text { Amazônia, Cerrado, Mata } \\
\text { Atlântica }\end{array}$ \\
\hline Dryopteridaceae & $\begin{array}{l}\text { C. meniscioides (Willd.) C. Presl Tent. } \\
\text { Pterid. } 85,1836 \text {. }\end{array}$ & Erva - Terrícola & $\begin{array}{l}\text { AC, AM, AP, RO, TO; BA, CE, PB, PE, PI; DF, GO, MS, MT; ES, MG, SP; SC } \\
\text { Nova Ocorrência para o Maranhão }\end{array}$ & $\begin{array}{l}\text { Amazônia, Cerrado, Mata } \\
\text { Atlântica }\end{array}$ \\
\hline Lindsaeaceae & $\begin{array}{l}\text { Lindsaea guianensis (Aubl.) Dryand. } \\
\text { Trans. Linn. Soc. London 3: } 421797 .\end{array}$ & Erva - Terrícola & AC, AM, PA, RO, RR; BA, CE, MA; DF, GO, MT; MG, SP; PR & $\begin{array}{l}\text { Amazônia, Cerrado, Mata } \\
\text { Atlântica }\end{array}$ \\
\hline Lomarionsidaceae & $\begin{array}{l}\text { Nephrolepis biserrata (Sw.) Schott Gen. } \\
\text { Fil., ad pl. } 3,1834 \text {. }\end{array}$ & Erva - Epífita & $\begin{array}{l}\text { AC, AM, AP, PA, RO, RR; AL, BA, CE, MA, PB, PE, PI, RN, SE; DF, MS, MT; } \\
\text { ES, MG, RJ, SP; PR, RS, SC }\end{array}$ & $\begin{array}{l}\text { Amazônia, Caatinga, Cerrado, } \\
\text { Mata Atlântica, Pantanal }\end{array}$ \\
\hline Lomantopsicaceac & $\begin{array}{l}\text { Nephrolepis rivularis (Vahl) Mett. Ex } \\
\text { Krug Bt. Jahrb. Syst. 24: 122, } 1897 .\end{array}$ & Erva - Epífita & AC, AM, AP, PA, RO, RR; AL, BA, CE, MA, PE; MT; ES, RJ, SP; PR, RS, SC & $\begin{array}{l}\text { Amazônia, Cerrado, Mata } \\
\text { Atlântica }\end{array}$ \\
\hline Lygodiaceae & $\begin{array}{l}\text { Lygodium venustum Sw. J. Bot. } \\
\text { (Schrader) } 1801 \text { (2): } 303,1803 \text {. } \\
\text { Lygodium volubile } \text { Sw. J. Bot. (Schrader) } \\
1801 \text { (2): } 304,1803 .\end{array}$ & $\begin{array}{l}\text { Erva liana - } \\
\text { Terrícola } \\
\text { Erva liana - } \\
\text { Terrícola }\end{array}$ & $\begin{array}{l}\text { AC, AM, AP, PA, RO, RR, TO; AL, BA, CE, MA, PB, PE, PI, RN, SE; DF, GO, } \\
\text { MS, MT; ES, MG, RJ, SP; PR, RS, SC } \\
\text { AC, AM, AP, PA, RO, RR, TO; AL, BA, CE, MA, PB, PE, RN, SE; DF, GO, MS; } \\
\text { ES, MG, RJ, SP; PR, RS, SC }\end{array}$ & $\begin{array}{l}\text { Amazônia, Caatinga, Cerrado, } \\
\text { Mata Atlântica, Pantanal } \\
\text { Amazônia, Caatinga, Cerrado, } \\
\text { Mata Atlântica }\end{array}$ \\
\hline Polypodiaceae & $\begin{array}{l}\text { Phlebodium aureum (L.) J. Sm. J. Bot. } \\
\text { (Hooker) } 4: 59,1841 \text {. }\end{array}$ & Éífita & $\begin{array}{l}\text { AM, PA, RR, TO; AL, BA, CE, MA, PB, PE; DF, GO, MS, MT; ES, MG, RJ, SP; } \\
\text { PR, RS, SC }\end{array}$ & $\begin{array}{l}\text { Amazônia, Cerrado, Mata } \\
\text { Atlântica }\end{array}$ \\
\hline \multirow{5}{*}{ Pteridaceae } & $\begin{array}{l}\text { Acrostichum aureum L. Sp. Pl. 2: 1069, } \\
1753 \text {. }\end{array}$ & Erva - Terrícola & A, CE, MA, PE, RN, SE; ES, N & $\begin{array}{l}\text { Cerrado, Mata Atlântica Nova } \\
\text { Ocorrência para o Cerrado }\end{array}$ \\
\hline & $\begin{array}{l}\text { Adiantum deflectens Mart. Icon. Pl. } \\
\text { Crypt. } 94,1834 .\end{array}$ & Erva - Terrícola & TO, Al, BA, CE, MA, PB, PE, PI; DF, GO, MS, MT; ES, MG, RJ, SP & Cerrado \\
\hline & $\begin{array}{l}\text { Ceratopteris pteridoides (Hook.) Hieron. } \\
\text { Bot. Jahrb. Syst. } 34 \text { (5): 561, } 1905 .\end{array}$ & Erva - Aquática & AM, PA; BA, CE, MA, PE; MS, MT; ES, MG, RJ, SP; PR, RS, SC & $\begin{array}{l}\text { Amazônia, Mata Atlântica, } \\
\text { Pantanal }\end{array}$ \\
\hline & Bull. Sci. Soc. Philom. Paris 8: 186, 1821 & Erva - Aquática & BA, MA, PE; MS, MT; ES, RJ, SP & $\begin{array}{l}\text { Pantanal Nova Ocorrência para } \\
\text { o Cerrado }\end{array}$ \\
\hline & $\begin{array}{l}\text { Pityrogramma calomelanos ((L.) Link } \\
\text { Handbuch 3:20 } 1833 \text {. }\end{array}$ & Erva - Terrícola & $\begin{array}{l}\text { AC, AM, AP, PA, RO, RR, TO; AL, BA, CE, MA, PB, PE, PI, RN, SE; DF, GO, } \\
\text { MS, MT; ES, MG, RJ, SP; PR, RS, SC }\end{array}$ & $\begin{array}{c}\text { Amazônia, Caatinga, Cerrado, } \\
\text { Mata Atlântica, Pampa, } \\
\text { Pantanal }\end{array}$ \\
\hline Salviniaceae & $\begin{array}{l}\text { Salvinia auriculata Aubl. Hist. Pl. } \\
\text { Guiane 2: } 969 \text {, pl. } 367,1775 \text {. }\end{array}$ & $\mathrm{E}$ & $\begin{array}{l}\text { AM, AP, PA, RR, TO; AL, BA, CE, MA, PB, PE, PI, RN, SE; DF, MS, MG; ES, } \\
\text { MG, RJ, SP; PR, RS, SC }\end{array}$ & $\begin{array}{l}\text { Amazônia, Cerrado, Mata } \\
\text { Atlântica, Pantanal }\end{array}$ \\
\hline Selaginellaceae & $\begin{array}{l}\text { Selaginella marginata (Humb. \& Bonpl. } \\
\text { Ex Willd.) Spring Flora } 21: 194,1838 .\end{array}$ & Erva - Rupícola & BA, MA, PI; DF, GO, MS, MT; MG, RJ, SP; PR, RS, SC & Cerrado, Mata Atlântica \\
\hline \multirow{3}{*}{ Thelypteridaceae } & $\begin{array}{l}\text { Meniscium serratum Cav. Descr. Pl. 548, } \\
1802 .\end{array}$ & Erva - Terrícola & AC, PA; AL, BA, MA, PE; GO, MS, MT; ES, MG, RJ, SP; PR, SC & Cerrado, Mata Atlântica \\
\hline & $\begin{array}{l}\text { Thelypteris interrupta (Willd.) K. Iwats. } \\
\text { J. Jap. Bot. 38(10): 38(10):314, } 1963 .\end{array}$ & Erva - Terrícola & $\begin{array}{l}\text { AM, AP; AL, BA, CE, MA, PB, PE; DF, GO, MS, MT; ES, MG, RJ, SP; PR, RS, } \\
\text { SC }\end{array}$ & $\begin{array}{l}\text { Amazônia, Cerrado, Mata } \\
\text { Atlântica }\end{array}$ \\
\hline & $\begin{array}{l}\text { Thelypteris reticulata (L.) Proctor Bull. } \\
\text { Inst. Jamaica, Sci. Ser. 5: } 63,1953\end{array}$ & Erva - Terrícola & $\begin{array}{l}\text { AM, AP; AL, BA, CE, MA, PB, PE; DF, GO, MS, MT; ES, MG, RJ, SP; PR, RS, } \\
\text { SC }\end{array}$ & $\begin{array}{l}\text { Amazônia, Cerrado, Mata } \\
\text { Atlântica }\end{array}$ \\
\hline
\end{tabular}


O baixo número de espécies pode ser confirmado pelos dados observados em outros trabalhos realizados no domínio fitogeográfico. No levantamento florístico de Zambiase et al. (2016), que realizaram em uma área de Cerrado de Goiás, catalogaram 40 espécies (36 espécies de Samambaias e 04 espécies de Licófitas). Da mesma forma, Lehn e Assis (2013), em um fragmento do Cerrado do Mato Grosso do Sul, listaram a ocorrência de 29 espécies e duas variedades (destas, apresentando uma únia espécie de Licófitas). Athayde-Filho e Felizardo (2007) realizaram um inventário florístico em três fragmentos no Cerrado Matogrossense, resultando em 25 espécies de Samambaias e uma espécie de Licófitas. Sendo assim, reforça-se a necessidade de estudos florísticos no Maranhão, pois Lehn e Assis (2013) ressaltam que a falta de levantamentos pteridofiticos fornecem impressões que a diversidade e riqueza de Samambaias e Licófitas estejam fracamente representadas na região.

\section{Conclusão}

A área estudada, apesar de sofrer ações antrópicas, apresenta comunidades de Monilophytas e Lycophytas, com um novo registro para o Maranhão, o que serve de indício para novas pesquisas, considerando a diversidade de espécies de pteridófitas para o Cerrado do Maranhão.

\section{Agradecimentos}

À Universidade Estadual do Maranhão/UEMA, Centro de Estudos Superiores de Caxias/CESC pelo apoio e infraestrutura disponibilizada para a pesquisa. À Fundação de Amparo à Pesquisa e ao Desenvolvimento Científico e Tecnológico do Maranhão/FAPEMA pelo apoio financeiro através do edital FAPEMA n ${ }^{\circ}$ 026/2013 Cbioma/Apoio ao Programa Acervos do Maranhão Coleções Biológicas e Arqueológicas.

\section{Referências Bibliográficas}

Athayde-Filho, F.P.; Felizardo, M.P.P. 2007. Florística e aspectos ecológicos da pteridoflora em três segmentos florestais ao longo do Rio Pindaíba, Mato Grosso. Pesquisas Botânica, São Leopoldo, 58: 227-244.

Bastos, C.C.C.; Cutrim, M.V.J. 1999. Pteridoflora da Reserva florestal do Sacavém, São Luís - Maranhão. Boletim do Museu Paraense Emílio Goeldi, série Botânica. 15(1): 3-37.

Conceição, G.M.; Rodrigues, M. S. 2010. Pteridófitas do Parque Estadual do Mirador, Maranhão, Brasil. Caderno de Geociências, 7: 47-53.

Conceição, G.M.; Ruggieri, A.C. 2010. Pteridófitas do Município de Tufilândia, Estado do Maranhão, Brasil. Pesquisa em Foco, 18(1): 59-68.

Costa, F.R.C. 2004. Structure and composition of the ground-herb community in a terra-firme Central Amazonian forest. Acta Amazonica. 34(1): 5359.

Fernandes, R.S.; Conceição, G.M.; Brito, E.S.; Paula-Zárate, E.L. 2007. Diversidade florística de pteridófitas da Área de preservação Ambiental do Inhamum, Caxias, Maranhão, Brasil. Revista Brasileira de Biociências. 5: 411-413.

Fernandes, R.S.; Conceição, G.M.; Costa, J.M.; Zárate, E.L.P. 2010. Samambaias e licófitas do município de Caxias, Maranhão, Brasil. Boletim do Museu Paraense Emílio Goeldi. Ciências Naturais, Belém, 5(3): 345-356.

Fidalgo, O.; Bononi, V.L.R. 1989. Técnicas de coleta, preservação e herborização de material botânico. Instituto de Botânica de São Paulo. p. 62 .

Flora do Brasil 2020. 2017. Jardim Botânico do Rio de Janeiro. Disponível em: < http://floradobrasil.jbrj.gov.br>. Acesso em: 20 Mai. 2016.

Forsthofer, M.; Athayde Filho, F.P. 2012. Florística e aspectos ecológicos de samambaias e licófitas ao longo do córrego Cachoeirinha, Nova Xavantina-MT. Pesquisas Botânica. 63: 149-164.

Goetz, M.N.B.; Fraga, L.L.; Schmitt, J.L. 2012. Florística e aspectos ecológicos de samambaias e licófitas em um parque urbano do Rio Grande do Sul, Brasil.

Gonzatti, F.; Valduga, E.; Wasum, R.; Scur, L. 2014. Florística e aspectos ecológicos de samambaias e licófitas em remanescentes de matas estacionais deciduais da serra gaúcha, Rio Grande do Sul, Brasil. Revista Brasileira de Biociências 12(2): 90-97.

IBGE. Satélite Maps: Município de São João do Sóter. 2012. Disponível em: $<$ http://cidades.ibge.gov.br/xtras/perfil.php?codmun=211107>. Acesso em: 20 Mai. 2016

Klink, C.A.; Machado, R.B. 2005. A conservação do Cerrado brasileiro. Megadiversidade. Belo Horizonte. 1(1): 148-155.

Kramer, K. U.; Green, P. S. 1990. Pteridophytes and Gymnosperms. In: Kubitzki, K. The families and Genera of Vascular Plants. Berlin, Springer-Verlag, p. 404.

Lehn, C.R.; Assis, E.L.M. 2013. Riqueza de samambaias e licófitas de uma mata de galeria na região central de Mato Grosso do Sul. Biotemas, 26(1): 7-15.

Mendonça, R.C.; Felfili, J.M.; Walter, B.M.T.; Silva Junior, M.C.; Rezende, A.V.; Filgueiras, T.S.; Nogueira, P.E.; Fagg, C.W. 2008. Flora vascular do Bioma Cerrado. Checklist com 12.356 espécies. In: Sano, S.M.; Almeida, S.P.; Ribeiro, J.F. Cerrado - ecologia e flora. Brasília, Embrapa Cerrados p. 423-1279.

Moran, R.C. 2008. Diversity, Biogeography, and Floristics. In: Ranker, T.A.; Haufler, C.H. Biology and evolution of ferns and lycophytes. Cambridge University Press, Cambridge. p. 367-394.

Prado, J. 1998. Reino Vegetal: Pteridophyta. In: FAPESP. Série Biodiversidade, São Paulo, cap. 5, p. 49-61.

Prado, J. 2005. Pteridaceae. In: Cavalcanti, T.B.; Ramos, A.E. Flora do Distrito Federal. Embrapa, Brasília. p. 185-215.

Prado, J.; Sylvestre, L.S. 2010. Introdução: as samambaias e licófitas do Brasil. In: Forzza, R. C. et al. Catálogo de plantas e fungos do Brasil [online]. Instituto de Pesquisas Jardim Botânico do Rio De Janeiro. Rio de Janeiro. 1: 69-74.

Pryer, K.M.; Schuettpelz, E.; Wolf, P.G.; Schneider, H.; Smith, A.R.; Cranfill, R. 2004. Phylogeny and evolution of ferns (Monilophytes) with a focus on the early leptosporangiate divergences. American Journal of Botany. 91. 10: 1582-1598.

Smith, A. R.; Pryer, K.M.; Schuettpelz, E.; Korall, P.; Schneider, H.; Wolf, P.G. 2006. A classification for extant ferns. Taxon. Utrecht, 55(3): 70531.

Smith, A.R.; Pryer, K.M.; Schuettpelz, E.; Korall, P.; Schneider, H.; Wolf, P.G. 2008. Fern classification. In: Ranker, T.A.; Haulfer, C.H. Biology and Evolution of Ferns and Lycophytes. Cambridge University Press. Cambridge. p. 417-461.

Windisch, P.G. 1992. Pteridófitas da Região Norte-Ocidental do Estado de São Paulo: guia para excursões. 2. ed. São José do Rio Preto: UNESP, p. 110.

Zambiase, R.M.; Resende, I.L.M.; Kreutz, C. Athayde Filho, F.P. 2016. Análise Ecoflorística de Samambaias e Licófitas em Mata de Galeria Inundável e Vereda, Quirinópolis, Goiás, Brasil. Pesquisas. Botânica. São Leopoldo: Instituto Anchietano de Pesquisas (69): 169-181.

Zuquim, G.; Costa, F.R.C.; Prado, J.; Tuomisto, H. 2008. Guia de samambaias e licófitas da REBIO Uatumã, Amazônia Central. Design Ed., Manaus. p.316. 